\title{
The Role of Enhanced External Counterpulsation Therapy in the Management of Coronary Artery Disease
}

\author{
Ozlem Soran \\ University of Pittsburgh, Heart and Vascular Institute
}

USA

\section{Introduction}

Coronary artery disease (CAD) is a narrowing or blockage of the arteries and vessels that provide oxygen and nutrients to the heart. It is caused by atherosclerosis, an accumulation of fatty materials on the inner linings of arteries. According to the American Heart Association and American Stroke Association's 2010 publication on heart disease and stroke statistics, cardiovascular disease remains the leading cause of mortality in the United States in men and women of every major ethnic group. It accounts for nearly 1 million deaths per year as of 2006 and was responsible for one in almost three deaths in the United States in 2006. Approximately 17 million persons have a history of coronary artery disease and 8 million have suffered a myocardial infarction (American Heart Association 2010). Medication and invasive revascularization are the most common approaches for treating CAD. Invasive revascularization includes percutaneus coronary interventions (PCI: stent implantation or balloon angioplasty)and Coronary Bypass Surgery (CABG). Even though both treatment options are commonly used, it is important to note that neither of these approaches provides a cure. In other words, although the symptoms are eliminated or alleviated, the disease and its causes are still present after treatment. Both treatments target lesions that cause the obstructions however, CAD is a progressive disease. New treatment approaches are in need to prevent the disease from progressing and the symptoms from recurring. Enhanced External Counterpulsation (EECP) therapy with its different mode of action provides a new treatment modality in the management of CAD and complements the invasive revascularization (Fig. 1).

This chapter summarizes the current evidence to support the role of EECP therapy in CAD management and provides information on its mechanism of action.

\section{What is EECP?}

EECP therapy consists of a treatment bed attached to an air compressor unit which is attached to a computerized control console. Three sets of cuffs wrapped around the lower legs and the buttocks of the patient. It is a noninvasive outpatient therapy consisting of electrocardiography (ECG)-gated sequential leg compression, which produces hemodynamic effects similar to those of an intra-aortic balloon pump (IABP) (Fig. 2). 


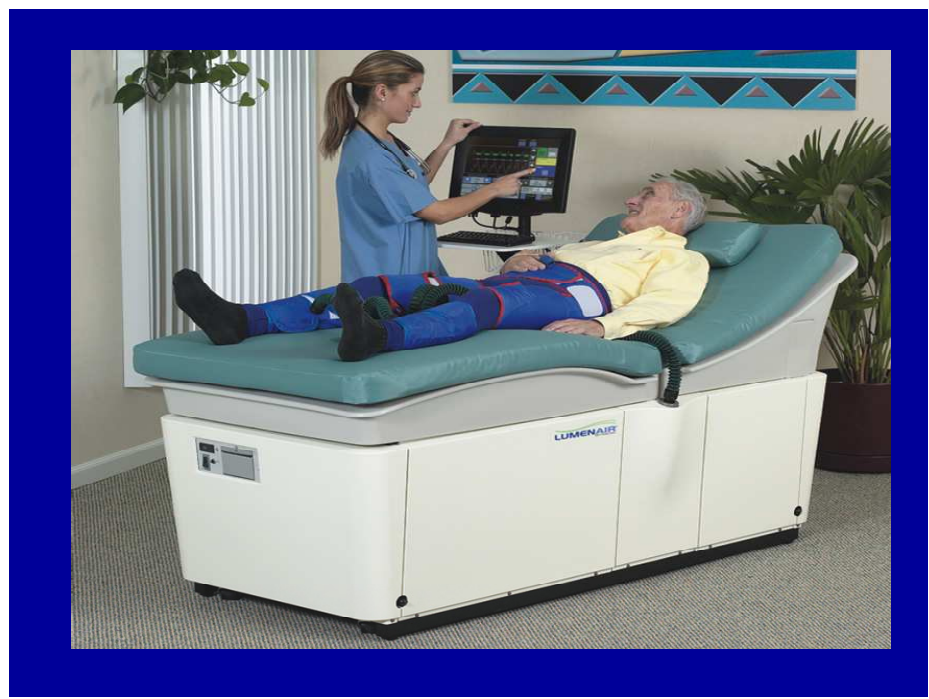

EECP Therapy: Consisting of a patient bed attached to an air compressor unit; computerized control consul; 3 sets of cuffs wrapped around the lower legs and the buttocks of the patient. (With permission from Vasomedical Inc., NY). EECP: Enhanced External Counterpulsation

\section{Fig. 1. EECP Therapy}
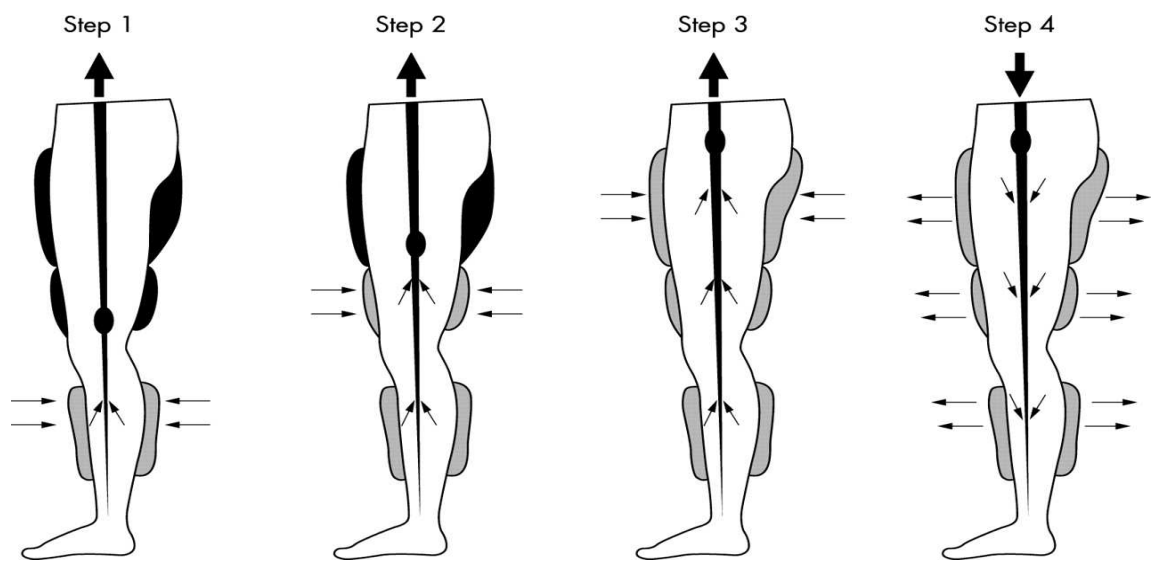

Three pairs of pneumatic cuffs are applied to the calves, lower thighs, and upper thighs. The cuffs are inflated sequentially during diastole, distal to proximal. The compression of the lower extremity vascular bed increases diastolic pressure and flow and increases venous return. The pressure is then released at the onset of systole. Inflation and deflation are timed according to the $\mathrm{R}$ wave on the patient's cardiac monitor. The pressures applied and the inflation-deflation timing can be altered by using the pressure waveforms and ECG on the EECP therapy monitor. (Adapted from Manchanda and Soran 2007)

ECG: Electrocardiogram; EECP: Enhanced External Counterpulsation

Fig. 2. Technique of EECP Therapy 
However, unlike IABP therapy, EECP therapy also increases venous return (Manchanda and Soran 2007, Birtwell et al 1968). Cuffs resembling oversized blood pressure cuffs - on the calves and lower and upper thighs, including the buttocks-inflate rapidly and sequentially via computer interpreted ECG signals, starting from the calves and proceeding upward to the buttocks during the resting phase of each heartbeat (diastole). This creates a strong retrograde counterpulse in the arterial system, forcing freshly oxygenated blood toward the heart and coronary arteries while increasing the volume of venous blood return to the heart under increased pressure. Just before the next heartbeat, before systole, all three cuffs deflate simultaneously, significantly reducing the heart's workload. This is achieved because the vascular beds in the lower extremities are relatively empty when the cuffs are deflated; significantly lowering the resistance to blood ejected by the heart and reducing the amount of work the heart must do to pump oxygenated blood to the rest of the body. A finger plethysmogram is used throughout treatment to monitor diastolic and systolic pressure waveforms. The current EECP device can generate external cuff pressures as high as 220 to $300 \mathrm{mmHg}$. A typical therapy course consists of 35 treatments administered for 1 hour a day over 7 weeks.

\subsection{Historical perspective}

The concept of counterpulsation was introduced in the U.S. in 1953 when Kantrowitz first proposed that elevations of aortic diastolic pressure could improve coronary blood flow and could benefit patients with coronary insufficiency. Although subsequent studies suggested that counterpulsation would indeed benefit some patients with coronary insufficiency, the development of the necessary technology proved challenging. A group of devices that increased coronary blood flow, including flow assist devices and venoarterial bypass systems, proved effective in providing increased coronary blood flow but were unable to reduce the tension time index or work load of the heart. External cardiac massage, internal cardiac massage, veno-arterial bypass, implantable auxillary ventricules, intra-aortic balloon pumps, and cardiopulmonary bypass have all demonstrated hemodynamic benefits; however, their usefulness in clinical practice has been limited because of their inherent invasiveness.

In the mid 1960s, several groups began to explore a non-invasive method for producing the salutary physiologic effects of counterpulsation. First external countrepulation device introduced by Harvard investigators, consisted of a hydraulically driven unit with a pair of water -filled bladders that could be wrapped around the lower legs and thighs of the patient. (Birtwell et al, 1968) However, the external pressure could only be applied to a limited amount of tissue mass thereby producing suboptimal diastolic augmentation of coronary flow. Investigators did not give up on the idea and kept working on to improve the technology. Over the years air-driven EECP system consisting of three sets of balloons wrapped around the lower legs, thighs and upper thighs of the patient has been developed. Air pressure was applied sequentially from the lower legs to the thigh and upper thigh resulting in "milking" of oxygenated blood from the lower extremities toward the heart with greater efficiency. (Amsterdam et al.,1980; Zeng et al., 1983). It has been demonstrated that the sequential inclusion of the upper thigh provided a critical advantage to EECP, affecting a $44 \%$ increase in diastolic augmentation compared with studies using only the lower extremity cuffs. Subsequent modifications of the EECP prototype with microprocessors allowed for precise cuff inflation and deflation and gating with the 
electrocardiogram. The lower cuffs were inflated at the start of diastole as represented by the beginning of the $\mathrm{T}$ wave, while simultaneous deflation of all three chambers was triggered just prior to systole at the onset of the $\mathrm{P}$ wave. The retrograde flow provided by EECP increased both the volume and pressure of diastolic flow such that diastolic to systolic ratios exceeded 1.0-1.2. (Soran et al., 1999). In summary, it took 40 years for investigators to develop the enhanced technology that is being currently used. (Fig 1)

\subsection{Acute hemodynamic effects of EECP therapy}

Acute hemodynamic effects of EECP has been assessed through both noninvasive and invasive techniques.(Soran et al., 2001; Stys et al., 2001; Michaelset al.,2001; Lakshmi et al. 2002; Nichols et al., 2006; Taguchi et al., 2004).

Taguchi et al, assessed the hemodynamic effects of EECP and compared it with IABP in 39 patients with uncomplicated acute myocardial infarction who underwent successful balloon coronary angioplasty within 12 hours after onset of chest pain. The radial artery and subclavian vein were cannulated to measure right atrial pressure, pulmonary capillary wedge pressure (PCWP), cardiac index, and systemic vascular resistance. Radial artery pressure tracing was used to measure area under artery pressure curves in systolic and diastolic phases. Sixty minutes treatment was administered to the patients in the EECP group. All parameters were measured before EECP, at 15, 30, 45, and 60 minutes after starting EECP, and 60 minutes after stopping EECP. After starting IABP support, measurements were obtained at 15,30,45, and 60 minutes. There were no significant changes in heart rate in either group before, during, and after treatments. Right atrial pressure increased significantly at 15 and 30 minutes after starting EECP, and then decreased gradually. There was no significant increase in right atrial pressure at 45 and 60 minutes after starting EECP compared with baseline. Right atrial pressure did not change in the IABP group, and there was no significant difference between 2 groups, except at 15 minutes after starting treatment. In the EECP group the PCWP significantly increased at 15 and 30 minutes, and then decreased gradually, but no significant change was seen in IABP group. Between two groups, there were no significant differences at baseline and during treatment. However, 60 minutes after stopping treatment, PCWP was significantly lower in the EECP group. The cardiac index in EECP group increased significantly at 45 and 60 minutes compared with the baseline, but no significant change was noted in IABP group. The increase in cardiac index at 60 minutes in the EECP group was significantly greater than that in the IABP group. Mean values of the area under the arterial pressure curves during the diastolic phase increased significantly in both groups, and there was no significant difference between two groups at any measuring point. Mean values of the areas under the artery pressure curves during the systolic phase decreased significantly during treatment compared with baseline in the IABP group. No significant change was observed in the EECP group. In both groups, systemic vascular resistance decreased significantly during treatment compared with baseline, but no significant difference was seen between two groups. This study showed that the hemodynamic effects of EECP were similar to those of IABP for diastolic augmentation and systemic vascular resistance. However, right atrial pressure, PCWP, and cardiac index increased during EECP in contrast to IABP. These effects suggest that EECP increases venous return, raises cardiac preload, and increases cardiac output. (Taguchi et al., 2004; Michaels et al., 2009) 
In another study, Taguchi et al., assessed the hemodynamic and neurohormonal changes during EECP. Sixty minutes of EECP therapy has been performed in patients with stable acute myocardial infarction who had undergone PCI. Right heart catheterization and neurohumoral parameters were assessed before, during 15, 30 and 60 minutes after EECP. Blood levels of atrial natriuretic peptide (ANP), brain (or B type) natriuretic peptide (BNP), renin, aldosterone, dopamine, and noradrenaline were assessed. Left ventricular ejection fraction and size were assessed invasively during the admission and between days 13 to 16 . Cardiac index increased from $3.3 \pm 0.8 \mathrm{~L} / \mathrm{min}$ before treatment to $4.1 \pm 0.8 \mathrm{~L} / \mathrm{min}$ at 60 minutes $(P<0.01)$. Right atrial pressure increased from $6.4 \pm 3.3 \mathrm{mmHg}$ at baseline to $9.8 \pm 4.0$ $\mathrm{mmHg}$ at 15 minutes $(P<0.01)$, and PCWP increased from $8.9 \pm 4.0 \mathrm{mmHg}$ to $12.6 \pm 5.3 \mathrm{mmHg}$ $(P<0.05)$. The blood levels of ANP increased from $54 \pm 42 \mathrm{pg} / \mathrm{ml}$ at baseline to $70 \pm 46 \mathrm{pg} / \mathrm{ml}$ at 60 minutes, which returned to baseline level 60 minutes after EECP treatment. The pretreatment concentrations of BNP, dopamine, noradrenaline, renin and aldosterone did not change during or after EECP treatment. Left ventricular end-diastolic pressure decreased from $18.6 \pm 1.6 \mathrm{mmHg}$ during the acute stage to $13.8 \pm 6.4 \mathrm{mmHg}$ during subacute follow-up, but there was no change in left ventricular ejection fraction or end-diastolic volume index. Blood concentrations of ANP increased at 15 minutes after initiation of EECP, which suggested that EECP treatment increased the volume of venous return, resulting in an increased atrial load. EECP significantly improved cardiac index with a significant increase in blood ANP concentration but without increase in heart rate or BNP. These findings suggest that there be an increased atrial preload without any significant change in ventricular preload.( Taguchi et al.,2004; Michaels et al., 2010)

Michaels et al., used an invasive approach to measure intracoronary, central aortic, and left ventricular pressure and intracoronary Doppler flow to assess the acute hemodynamic effects of EECP. Ten patients who were referred for cardiac catheterization ( 5 with suspected coronary artery disease, 3 with severe mitral regurgitation, and 2 with heart transplant patients) were included in the study. Key exclusion criteria included severe aortic insufficiency, decompensated heart failure, significant arrhythmia, systolic blood pressure more than $180 \mathrm{mmHg}$, and symptomatic peripheral arterial disease. EECP was performed at external cuff pressures ranging from 100 to $300 \mathrm{mmHg}$. At baseline and during EECP, simultaneous central aortic and intracoronary pressure, intracoronary Doppler flow velocity, and corrected thrombosis in Myocardial Infarction study (TIMI) frame count (CTFC) were measured. Peak aortic systolic pressure decreased $11 \%$ from $114 \pm 19 \mathrm{mmHg}$ at baseline to $101 \pm 28 \mathrm{mmHg}$ during $\mathrm{EECP}(P=0.02)$. These findings indicate that EECP acutely reduces ventricular afterload. Left ventricular end-diastolic pressure decreased during EECP $(15 \pm 7 \mathrm{mmHg}$ at baseline, $13 \pm 6 \mathrm{mmHg}$ during EECP; $P=0.17)$. Aortic diastolic pressure increased $92 \%$ from $71 \pm 10 \mathrm{mmHg}$ to $136 \pm 22 \mathrm{mmHg}(P<0.0001)$, and mean aortic pressure increased $16 \%$ from $88 \pm 10 \mathrm{mmHg}$ to $102 \pm 14 \mathrm{mmHg}$ during EECP $(P=0.0007)$, indicating significant. Intracoronary pressure was measured in an unobstructed epicardial coronary artery using the micromanometer-tipped 0.014 -inch pressure wire. There was a $93 \%$ increase in intracoronary peak diastolic pressure $(71 \pm 10 \mathrm{mmHg}$ at baseline to $137 \pm 21 \mathrm{mmHg}$ during EECP; P<0.0001;. (Michaels et al., 2002; Fig 3)

Mean pressure increased by $16 \%$ from $88 \pm 9 \mathrm{mmHg}$ at baseline to $102 \pm 16 \mathrm{mmHg}$ during EECP $(P=0.006)$. EECP decreased peak systolic pressure by $15 \%$ from $116 \pm 20 \mathrm{mmHg}$ at baseline to $99 \pm 26 \mathrm{mmHg}$ during EECP $(P=0.002)$. Planimetry of the intracoronary pressure tracings showed a $28 \%$ increase in diastolic pressure $(42 \pm 9 \mathrm{mmHg}$ sec at baseline; $54 \pm 15 \mathrm{mmHg}$ sec during EECP; $P=0.0003$ ) and a $12 \%$ decrease in systolic pressure 

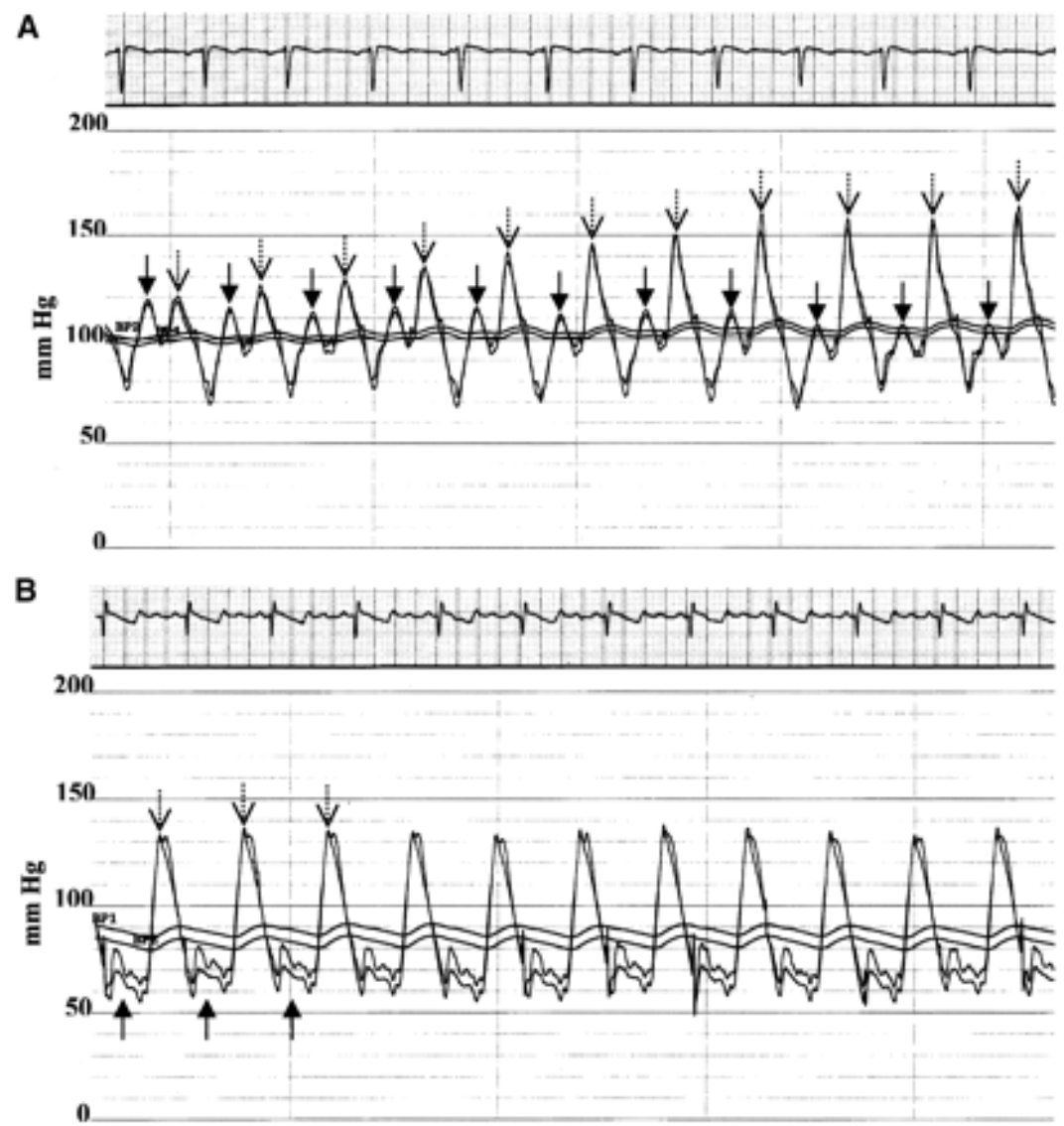

Representative simultaneous hemodynamic tracings of central aortic pressure from the coronary catheter and intracoronary pressure from the PressureWire. In tracings of both phasic and mean pressure (bottom) obtained at the beginning of EECP (A), there is a gradual increase in peak diastolic (dashed arrows) and mean pressure with a decrease in peak systolic pressure (solid arrows) attributable to systolic unloading as the inflation pressure is increased in the EECP device. In another patient, diastolic augmentation is demonstrated during EECP at a cuff pressure of $300 \mathrm{~mm} \mathrm{Hg}$ (B). The intracoronary coronary pressure was $5 \mathrm{~mm} \mathrm{Hg}$ lower than central aortic pressure, attributable to diffuse coronary atherosclerosis. The paper speed is $25 \mathrm{~mm} / \mathrm{sec}$. ( With permission from Michaels AD,2002)

Fig. 3. Hemodynamic tracings of central aortic pressure

( $33 \pm 6 \mathrm{mmHg}$ sec at baseline; $29 \pm 7 \mathrm{mmHg}$ sec during EECP; $P=0.008$ ). Intracoronary Doppler flow velocity was measured using a 0.014-inch FloWire (Fig 2). The average peak velocity increased $109 \%$ from $11 \pm 5 \mathrm{~cm} / \mathrm{s}$ at baseline to $23 \pm 5 \mathrm{~cm} / \mathrm{s}$ during EECP $(P=0.001)$. The peak diastolic velocity increased $150 \%$ from $18 \pm 7 \mathrm{~cm} / \mathrm{s}$ at baseline to $45 \pm 14 \mathrm{~cm} / \mathrm{s}$ during EECP $(P=0.0004)$. The diastolic-to-systolic velocity ratio increased $100 \%$ from $1.0 \pm 0.3$ to $2.0 \pm 0.7$ during EECP $(P=0.003)$. There was no significant change in peak systolic velocity. The CTFC, another measure of coronary flow velocity, significantly decreased $28 \%$ from $37 \pm 18$ at baseline to $27 \pm 13$ during EECP $(P=0.001)$. This study provides solid evidence of the increase 
in directly measured aortic and coronary diastolic pressure and flow velocity from EECP. There is a significant reduction in left ventricular afterload and left ventricle work secondary to systolic unloading.

(Michaels et al., 2010)

Acute hemodynamic effects of EECP can be summarized as: (Fig 4)

1. Increased retrograde aortic blood flow; diastolic augmentation;

2. Increased coronary blood flow ; increased perfusion pressure;

3. Increased venous return;

4. Increased cardiac output;

5. Systolic unloading;

6. Decreased left ventricular workload

\section{EECP Hemodynamic Effects}
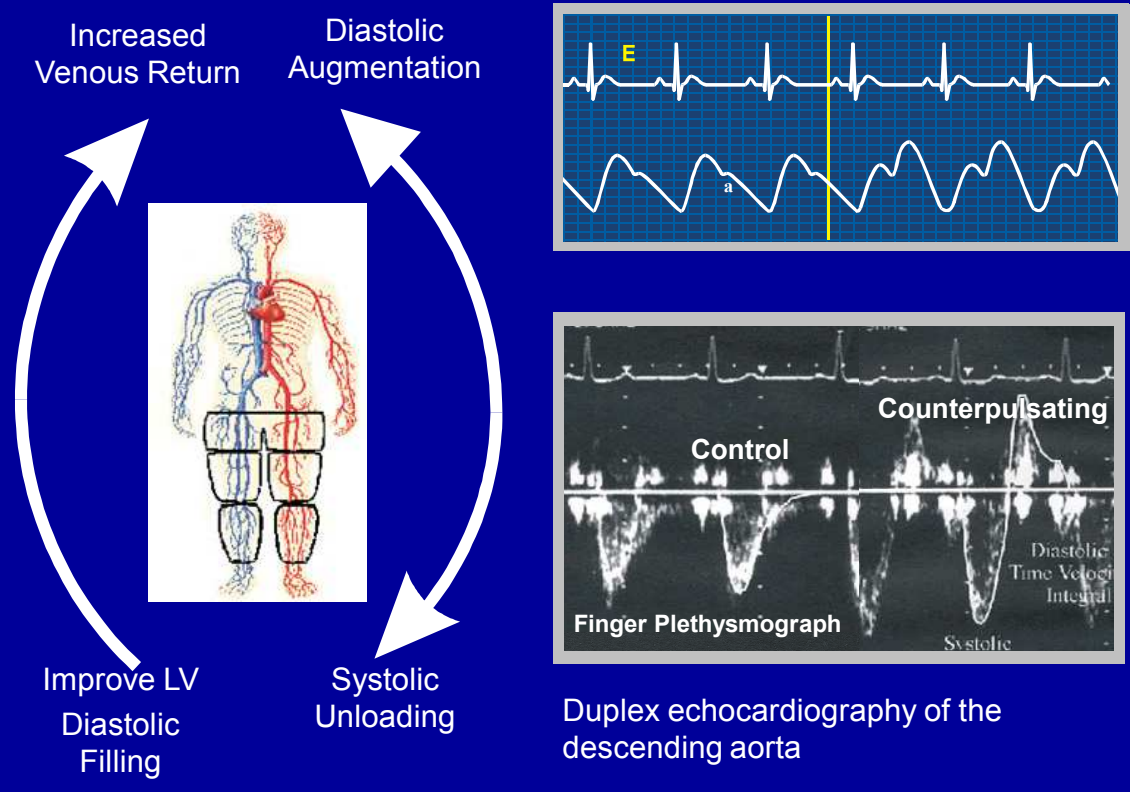

Duplex echocardiography of the descending aorta

Right upper corner: Showing the ECG tracing and finger pletysmogram. As soon as the device turned on (yellow line) diastolic augmentation starts. Right lower corner: showing the diastolic augmentation by echocardiography. EECP: Enhanced External Counterpulsation; a: aortic notch (With permission from Dr Soran and Katz 1998)

Fig. 4. Acute hemodynamic effects of EECP Therapy 


\section{EECP therapy in CAD management}

Several nonrandomized and randomized trials have demonstrated a consistently positive clinical response among patients with CAD treated with EECP (Lawson et al. 1992, Arora et al. 1999). Benefits associated with EECP therapy include reduction in angina and nitrate use, increased exercise tolerance, favorable psychosocial effects, and enhanced quality of life as well as prolongation of the time to exercise-induced ST-segment depression and an accompanying resolution of myocardial perfusion defects. Numerous clinical trials in the past two decades have shown EECP therapy to be safe and effective for patients with CAD, with a clinical response rate averaging $70 \%$ to $80 \%$, which is sustained up to 5 years. (Lawson et al. 2000; Masuda et al. 2001; Stys et al. 2002; Pettersson et al.2006; Braverman, DL. 2009).

Most studies on EECP therapy cannot be double blind and lack good control groups because of technical limitations, drawbacks that have frequently raised questions regarding operator bias. However, the Multicenter Study of EECP (MUSTEECP), a randomized double-blinded placebo-controlled trial, did document a clinical benefit from EECP therapy in patients with chronic stable angina and positive exercise stress tests. In this study, 139 patients (mean age 63 years, range 35 years to 81 years) with angina pectoris (typical Canadian Cardiovascular Society (CCS) classes I, II, and III angina) and documented coronary ischemia were equally randomized to hemodynamically inactive counterpulsation with EECP versus active counterpulsation. Patients in the active EECP therapy group showed a statistically significant increase in time to exercise-induced ST-segment depression when compared with sham and baseline, and reported a statistically significant decrease in the frequency of angina episodes when compared with sham and baseline. Exercise duration increased significantly in both groups; however, the increase was greater in the active EECP group. Moreover, a MUST-EECP sub study demonstrated a significant improvement in quality-of life parameters in patients assigned to active treatment, and this improvement was sustained during a 12-month follow-up (Arora et al. 2002).

Tartaglia et al assessed the effect of EECP on exercise capacity and myocardial perfusion by comparing results of maximal exercise radionuclide testing pre- and post-EECP treatment. This prospective study included 25 patients with angina who had performed maximal symptom-limited exercise tolerance tests with Bruce protocol and radionuclide perfusion single-photon emission computed tomography (SPECT) study prior to and at completion of EECP treatment. After $35 \mathrm{~h}$ of EECP, 93\% improved by at least one functional angina class. There is a significant improvement in their total treadmill times and a significant change in their peak double products, from $18,891+/-3,939$ pre-EECP to $20,464+/-4,305$ post-EECP exercise tolerance tests $(\mathrm{p}<0.03)$. Pre EECP, 16 patients had ST-segment depression on their initial exercise tolerance tests. After EECP, 80\% either no longer had ST depression or had a significant increase in their time to ST depression. The radionuclide perfusion scores also showed a significant reduction in ischemic segments. In this study, patients treated with EECP demonstrated a reduction in angina symptoms, improvement in exercise capacity, increase in time to ST-segment depression, and decrease in perfusion defects despite performing at a higher workload.

In another study, investigators evaluated the effects of EECP therapy on dobutamine stressinduced wall motion score among patients with angina pectoris. In this prospective study, $43 \%$ of patients with severe chronic angina pectoris and had a positive dobutamine stress echocardiogram had normal or reduced dobutamine induced wall motion abnormalities after EECP therapy. (Bagger et al., 2004) 
British investigators assessed the immediate and long-term effect of EECP in treatment of chronic stable refractory angina. Sixty-one patients were treated with EECP and 58 completed a course of treatment. About $52 \%$ of patients suffered from CCS III and IV angina prior to EECP. Immediately post-EECP, angina improved by at least one CCS class in $86 \%$ and by two classes in 59\%. At 1-year follow-up, sustained improvement in CCS was observed in $78 \%$ of the patients. The median weekly angina frequency and nitroglycerin use were significantly reduced immediately after EECP. In 48 patients, their mean exercise time improved significantly after EECP. Major-adverse events were rare. This study showed that for patients who fail to respond to conventional measures, a high proportion gain symptomatic benefit from EECP.

In 2006, Swedish investigators assess the long-term outcome of EECP treatment at a Scandinavian centre, in relieving angina in patients with chronic refractory angina pectoris. 55 patients were treated with EECP. CCS class, antianginal medication and adverse clinical events were collected prior to EECP, at the end of the treatment, and at six and 12 months after EECP treatment. Clinical signs and symptoms were recorded. EECP treatment significantly improved the CCS class in $79 \%$ of the patients with chronic angina pectoris. The reduction in CCS angina class was seen in patients with CCS class III and IV and persisted 12 months after EECP treatment. In accordance with the reduction in CCS classes there was a significant decrease in the weekly nitroglycerin usage. The results showed that EECP was a safe and effective treatment in CAD patients and the beneficial effects were sustained during a 12-months follow-up period. (Pettersson et al.,2006).

Same year, Italian investigators tested efficacy of EECP on symptoms, myocardial ischaemia and cardiac performance in patients with intractable angina, refractory to surgical and medical treatment. Twenty-five patients (mean age 65 years) with persistent ischaemia notwithstanding optimal medical therapy or after interventional or surgical procedure, received EECP sessions for $35 \mathrm{~h}$. Each patient underwent dobutamine stress echocardiography before and after treatment. Eighty-four percent of patients showed an increase in at least one functional angina class. Thirty-six percent of patients had a reduction in the area of inducible ischaemia at dobutamine stress echocardiography after treatment. They concluded that EECP therapy was effective in relieving symptoms in patients with refractory angina and may reduce inducible ischaemia at dobutamine stress echocardiography, especially in patients with reduced systolic function and compromised segmental kinesis. (Novo et al., 2006)

Although randomized (including placebo-controlled) and nonrandomized studies have shown beneficial effects of EECP therapy, investigators saw the need to assess EECP's effectiveness in real-world settings, leading them to develop the International EECP Patient Registry (IEPR) under the management of the University of Pittsburgh (Michaels et al. 2004, Soran et al., 2010). More than 5000 patients were enrolled in phase I and another 2500 patients enrolled in phase II of the study, and more than 90 centers participated. Results from the IEPR and the EECP Clinical Consortium have demonstrated that the symptomatic benefit observed in controlled studies translates to the heterogeneous patient population seen in clinical practice. Moreover, follow-up data indicate that the clinical benefit may be maintained for up to 5 years in patients with a favorable initial clinical response (Lawson et al. 2000; Loh et al 2008)

Soran et al., compared the efficacy, repeat EECP and 6-months major adverse cardiovascular events (MACE: Death/CABG/PCI/MI) free survival rates for patients treated with EECP for angina management in Europe (EU) with the United States (US); 4658 were treated and 
followed in the US and 262 in EU. EU were younger $(p<0.001)$ with a higher proportion of men. Previous revascularization rate was higher in US $(p<0.001)$. EU had less diabetes, hypertension, hyperlipidemia, multivessel disease, Class IV angina, and higher rates of nitroglycerin usage $(\mathrm{p}<0.001)$. After a mean treatment course of 34 hours, both groups showed a significant reduction in the severity of angina (78\% vs $76 \%)$. Discontinuation of nitroglycerin usage was similar in both groups $(50 \%)$. MACE during the treatment period was low in both groups $(<3 \%)$.Compliance with the treatment course was better in EU $(\mathrm{p}<0.001)$.

At 6-month follow up $66 \%$ of EU and $76 \%$ of US had maintained the improvement in angina class; survival rate was $99 \%$ in EU and $97 \%$ in US. MACE free survival rate was $92 \%$ in EU vs $90 \%$ in US. Repeat EECP rates at 6months follow up were significantly lower in EU $(0.5 \%$ vs. $4 \%, \mathrm{p}<0.01)$.Patients presenting for EECP treatment from EU and US populations showed very different baseline profiles. However, both cohorts achieved substantial reduction in angina with high event free survival rates at 6 months. (Soran et al., 2010)

EECP was suggested as a safe treatment option for selected symptomatic PCI candidates with obstructive CAD. Baseline characteristics and 1-year outcome in 2 cohorts of PCI candidates presenting with stable symptoms: 323 patients treated with EECP in the IEPR, and 448 NHLBI Dynamic Registry patients treated with elective PCI, were compared. Compared with patients receiving PCI, IEPR patients had a higher prevalence of many risk factors including prior PCI prior coronary artery bypass grafting, prior myocardial infarction, history of congestive heart failure, and history of diabetes. Left ventricular ejection fraction was lower among IEPR patients (mean $50.3 \%$ vs $59.2 \%, p<0.001$ ). At 1 year, survival was comparable in the 2 cohorts ( $98.7 \%$ IEPR vs $96.8 \% \mathrm{PCI}, \mathrm{P}=\mathrm{NS}$ ), as were rates of coronary artery bypass grafting during follow-up (4.5\% IEPR vs $5.7 \% \mathrm{PCI}, \mathrm{P}=\mathrm{NS})$. At 1 year, $43.7 \%$ of IEPR patients reported no anginal symptoms compared with $73.4 \%$ of Dynamic Registry patients ( $p<0.001$ ). Rates of severe symptoms (CCS class III, IV, or unstable) at 1 year were $15.5 \%$ among IEPR patients and $9.5 \%$ in the Dynamic Registry ( $\mathrm{p}=$ 0.02). PCI candidates suitable for and treated with EECP had 1-year major event rates comparable to patients receiving elective PCI. These results suggested that EECP, as a noninvasive treatment, could be used as a first -line treatment with invasive revascularization reserved for EECP failures, or high -risk patients. (Holubkov et al., 2002). The results of this study warrant the initiation of a randomized controlled study to ascertain the efficacy of EECP combined with drug therapy as a first line treatment in selected patients with CAD.

\subsection{EECP therapy in CAD with left ventricular dysfunction}

When providing EECP therapy to patients with heart failure, the initial researchers were concerned primarily that the increased venous return resulting from EECP therapy might precipitate pulmonary edema in angina patients with severe left ventricular dysfunction (SLVD).

Using outcomes data from 363 patients enrolled in the IEPR, Soran et al. (Soran et al. 2006, Soran et al. 2002) evaluated the safety and efficacy of EECP therapy in those with refractory angina pectoris and SLVD (ejection fraction $[\mathrm{EF}]<35 \%$ ). In this study patients' average duration of clinical CAD was nearly 13 years; $84 \%$ had multivessel disease and $93 \%$ were not candidates for further revascularization due to the extent and severity of disease, LV dysfunction, co-morbid conditions, previous interventions, or risk/benefit ratio. Angina was severe (class III/IV) in $93 \%$ of patients. There was a high prevalence of cardiac risk factors 
(i.e., $45 \%$ had DM, $85 \%$ had prior MI, ,78\% had hyperlipidemia, $77 \%$ had a history of smoking and $82 \%$ had a family history of premature atherosclerotic cardiovascular disease). More than $50 \%$ reported quality of life as poor. On average, patients underwent an EECP treatment course of 32 hours, with $81 \%$ completing the course. Twelve percent discontinued due to a clinical event, and $7 \%$ stopped due to patient preference. Major adverse cardiovascular events that occurred over the course of EECP therapy were low (Death/ myocardial infarction $[\mathrm{MI}] / \mathrm{CABG} / \mathrm{PCI}<2 \%)$. After completion of treatment, there was a significant reduction in angina severity: $72 \%$ improved from severe angina to no or mild angina. Fifty-two percent of the patients stopped using nitroglycerin. There was also a significant increase in quality of life. At 2-year follow-up, angina reduction was maintained in $55 \%$ of patients, the survival rate was $83 \%$, and event-free (death/MI/PCI/CABG)

\section{Death/Ml/CABG/PCl}

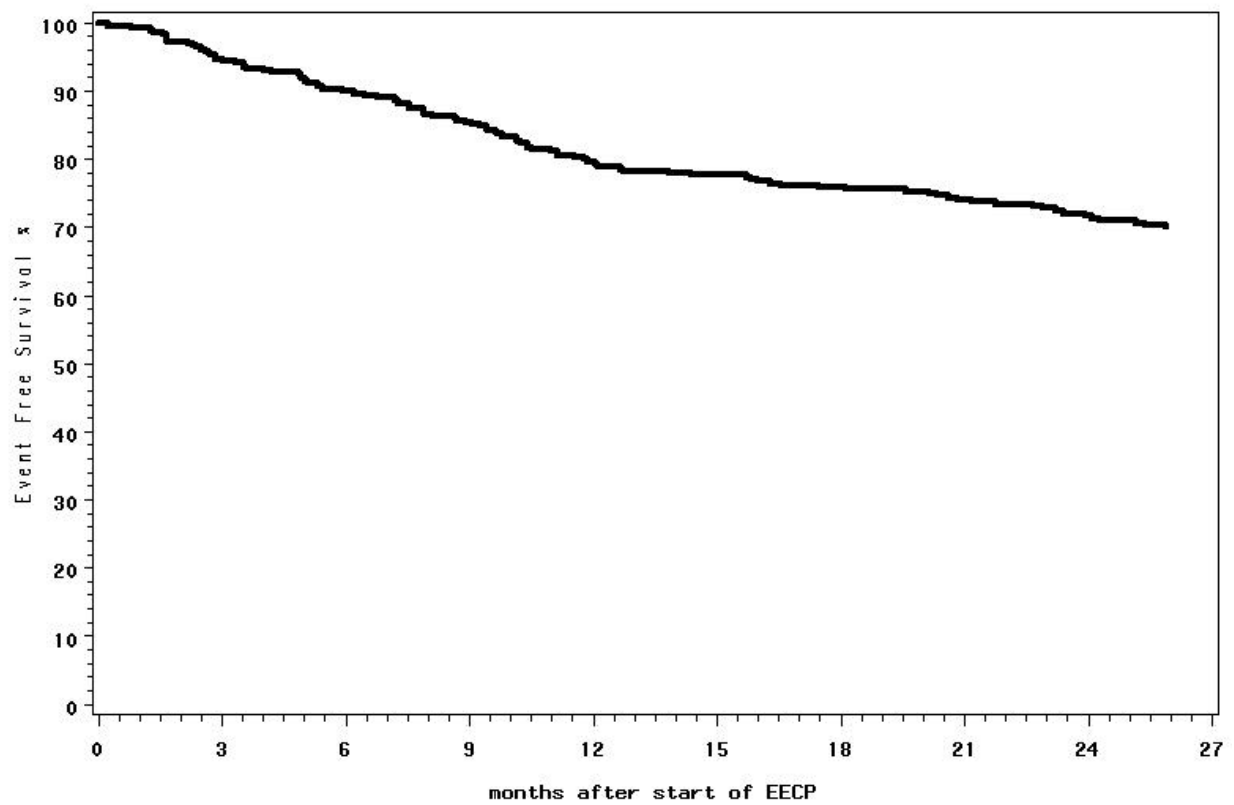

Event (CABG; PCI; MI) free survival rate at 2 years post EECP therapy in patients with coronary artery disease and SLVD (With permission from Dr. Soran; Soran et al 2006)

CABG: Coronary Artery Bypass Grafting Surgery, MI: Myocardial Infarction, PCI: Percutaneaus Coronary Intervention, SLVD: Severe left ventricular dysfunction

Fig. 5. Event Free Survival Rate at 2 years -Post EECP 
survival was $70 \%$. Forty-three percent had no cardiac hospitalization; $81 \%$ had no congestive heart failure event (Fig.5). They concluded that EECP therapy for angina is safe and effective in patients with SLVD who are not considered good candidates for revascularization by CABG or PCI.

Estahbanaty et al., evaluated the effects of EECP on systolic and diastolic cardiac function using echocardiography to measure left ventricular $\mathrm{EF}$, end-systolic volume, end-diastolic volume, systolic wave (Sm), early diastolic wave (Ea), Vp, E/Ea, E/Vp, and diastolic function grade in 25 patients before and after 35 hours of EECP. EECP reduced end-systolic volume and end-diastolic volume and increased EF significantly in patients with baseline left ventricular $\mathrm{EF} \leq 50 \%$, baseline $\mathrm{E} / \mathrm{Ea} \geq 14$, baseline grade II or III diastolic dysfunction (decreased compliance), baseline Ea $<7 \mathrm{~cm} / \mathrm{s}$ and baseline $5 \mathrm{~m}<7 \mathrm{~cm} / \mathrm{s}$. These results demonstrated improved systolic and diastolic function in selected patients. (Estahbanaty et al., 2007)

Lawson et al. evaluated CAD patients with preserved left ventricular function (PLV; EF > $35 \%)$ and with SLVD (EF $\leq 35 \%)$ who were treated with a 35-hour course of EECP. Bioimpedance measurements of cardiovascular function were obtained before the first and after the 35th hour of EECP therapy. Twenty-five patients were enrolled, 20 with PLV and 5 with SLVD. Angina class improved similarly in both groups. The SLVD group, in contrast to the PLV group, had increased cardiac power (ie, mean arterial pressure $\times$ cardiac output/451), stroke volume, and cardiac index and decreased systemic vascular resistance with treatment. This study suggests that EECP may benefit patients experiencing CAD with SLVD directly by improving cardiac power and indirectly by decreasing systemic vascular resistance (Lawson et al. 2002).

Patients with CAD and left ventricular dysfunction exert an enormous burden on health care resources, primarily because of the number of recurrent emergency department visits and hospitalizations. Improvements in symptoms and laboratory assessments in these patients may not correlate with a reduction in emergency room visits and hospitalizations. Soran et al. assessed the impact of EECP therapy on emergency department visits and hospitalization rates at 6-month follow up. In this prospective cohort study, clinical outcomes, number of all-cause emergency department visits, and hospitalizations within the 6 months before EECP therapy were compared with those at 6-month follow-up. The mean number of emergency department visits per patient decreased from $0.9 \pm 2.0$ before EECP to $0.2 \pm 0.7$ at 6 months $(P<0.001)$, and hospitalizations were reduced from $1.1 \pm 1.7$ to $0.3 \pm 0.7$ $(P<0.001)$ (Soran et al. 2007).

Scientific data indicates that EECP provides a safe and effective treatment option for patients with CAD and left ventricular dysfunction.

\section{Mechanism of action}

Upon diastole, cuffs inflate sequentially from the calves, raising diastolic aortic pressure proximally and increasing coronary perfusion pressure. Compression of the vascular beds of the legs also increases venous return. Instantaneous decompression of all cuffs at the onset of systole significantly unloads the left ventricle, thereby lowering vascular impedance and decreasing ventricular workload. This latter effect, when coupled with augmented venous return, raises cardiac output. In summary, EECP therapy increases venous return, raises cardiac preload, increases cardiac output, and decreases systemic vascular resistance (Michaels et al. 2002). 
Mechanism of action of EECP therapy is not through one pathway, but several pathways affecting each other. (Fig.6)

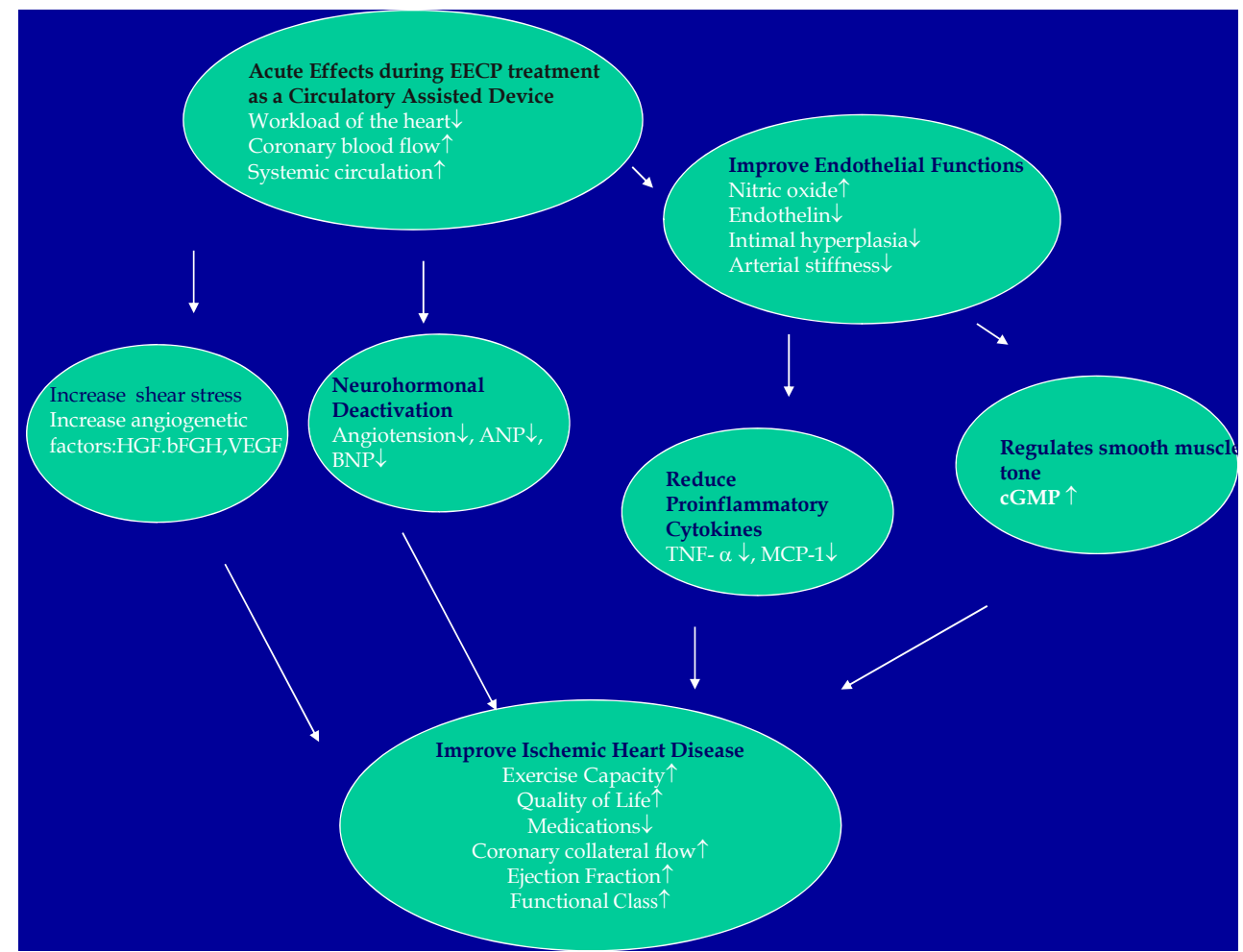

Possible mechanisms responsible for the clinical benefit associated with EECP therapy. Acute hemodynamic and neurohormonal changes, prolonged stimulus to increase sheer stress which promotes endothelial function improvement is thought to promote myocardial collateralization via opening of latent conduits, arteriogenesis, and angiogenesis.

HGF: hepatocyte growth factor, bFGF: basic Fibroblast Growth Factor, VEGF: Vascular endothelial growth factor, TNF: Tumor necrosis factor-alpha, MCP-1: Monocyte chemotactic protein-1, cGMP: Cyclic guanosine monophosphate

Fig. 6. Mechanism of EECP Therapy

\subsection{Collateral development}

One of the mechanisms of action is through collateral development. Mode-of-action studies have shown that EECP therapy increases angiogenesis factors such as human growth, basic fibroblast growth, and vascular endothelial growth factors. Enhanced diastolic flow increases shear stress, increased shear stress activates the release of growth factors, and augmentation of growth factor release activates angiogenesis.(Soran et al., 1999)

To clarify the mechanism of action of EECP therapy, Masuda et al used (13) N-ammonia positron emission tomography to evaluate myocardial perfusion. Eleven patients (mean age: 61 years) with angina pectoris underwent EECP therapy for $351 \mathrm{~h}$ sessions. Treadmill 
exercise test and (13) N-ammonia positron emission tomography, both at rest and with dipyridamole, before and after EECP therapy were performed. Neurohumoral factors and nitric oxide were also evaluated. Myocardial perfusion increased significantly at rest after therapy. In ischemic regions, particularly the anterior region, myocardial perfusion at rest and with dipyridamole and coronary flow reserve improved significantly after therapy. Exercise time was prolonged and the time to 1-mm ST depression improved markedly. After therapy, nitric oxide levels increased significantly and neurohumoral factors decreased. These results suggest that one of the mechanisms of the therapy is development and recruitment of collateral vessels. (Masuda et al., 2001).

To test the hypothesis that EECP augments collateral function Gloekler et al randomized patients with chronic stable angina to EECP therapy or sham treatment. Before and after 30 $h$ of randomly allocated EECP or sham EECP, the invasive collateral flow index (CFI) was obtained in 34 vessels. CFI was determined by the ratio of mean distal coronary occlusive pressure to mean aortic pressure with central venous pressure subtracted from both. Additionally, coronary collateral conductance (occlusive myocardial blood flow per aortocoronary pressure drop) was determined by myocardial contrast echocardiography and brachial artery flow-mediated dilatation was obtained. CFI significantly improved in the EECP group but not in the sham treatment. EECP appeared to be effective in promoting coronary collateral growth. The extent of collateral function improvement was related to the amount of improvement in the systemic endothelial function (Gloekler et al. 2010).

\subsection{Endothelial function improvement}

Another mechanism of action of EECP therapy is through endothelial changes. EECP therapy improves endothelial function and enhances vascular reactivity. Akhtar et al examined the effects of EECP on plasma nitric oxide and endothelin-1 (ET-1) levels. Plasma nitrate and nitrite (NOx) and ET-1 levels were measured serially in 13 patients with coronary artery disease who received 1-hour daily treatments of EECP over 6 weeks. During the course of EECP therapy, plasma NOx progressively increased and plasma ET-1 progressively decreased. After 36 hours of EECP, there was a $62 \%$ increase in plasma NOx compared with baseline ( $p$ $<0.0001$ ) and a $36 \%$ decrease in plasma ET-1 ( $\mathrm{p}<0.0001$ ). At 3 months after completion of EECP, NOx remained $12 \%$ above baseline $(p=0.002)$, and ET- 1 remained $11 \%$ below baseline $(p=0.0068)$. This data provides neurohormonal evidence to support the hypothesis that EECP improves endothelial function.(Akhtar Am J Cardiol 2006)

Braith et al investigated the effects of EECP on peripheral artery flow-mediated dilation in a randomized placebo-controlled study. Symptomatic patients with CAD were randomized (2:1 ratio) to thirty-five 1-hour sessions of either EECP or sham EECP . Flow-mediated dilation of the brachial and femoral arteries was performed with the use of ultrasound. Plasma levels of nitrate and nitrite, 6-ketoprostaglandin, F1a, endothelin-1, asymmetrical dimethylarginine, tumor necrosis factor- $\alpha$, monocyte chemoattractant protein- 1 , soluble vascular cell adhesion molecule, high-sensitivity C-reactive protein, and 8-isoprostane were measured. EECP significantly increased brachial and femoral artery flow-mediated dilation, the nitric oxide turnover/production markers nitrate and nitrite and 6-keto-prostaglandin F1a, whereas it decreased endothelin- 1 and the nitric oxide synthase inhibitor asymmetrical dimethylarginine in treatment versus sham groups, respectively. EECP significantly decreased the proinflammatory cytokines tumor necrosis factor- $\alpha$, monocyte chemoattractant protein-1, soluble vascular cell adhesion molecule-1, high-sensitivity Creactive protein, and the lipid peroxidation marker 8-isoprostane in treatment versus sham 
groups, respectively. EECP also significantly reduced angina classification in treatment versus sham groups, respectively. Findings from this study provide novel mechanistic evidence that EECP has a beneficial effect on peripheral artery flow-mediated dilation and endothelial-derived vasoactive agents in patients with symptomatic CAD (Braith et al. 2010). It is known that cGMP regulates vascular smooth muscle tone that may improve arterial function. French investigators assessed the effect of a single session of EECP on plasma and platelet Cyclic GMP (cGMP) in asymptomatic subjects with cardiovascular risk factors and in patients with CAD. Fifty-five subjects were randomized into two groups to receive either sham (control) or active EECP during $1 \mathrm{~h}$. Plasma and platelet cGMP were measured immediately before and after EECP by radioimmunoassay. One hour of EECP increased cGMP plasma concentration by $52 \% \pm 66 \%(\mathrm{SD})(\mathrm{P}<.001)$ and platelet content by $19 \% \pm 28 \%$ $(\mathrm{P}<.01)$. The increase in plasma cGMP was particularly marked in CAD patients receiving active EECP $(\mathrm{P}<.01)$, mainly in those with low LDL cholesterol. Platelets, inhibition of nitric oxide synthesis by NG-monomethyl-L-arginine (L-NMMA) reduced cGMP by $23 \% \pm 31 \%$ ( $P$ _. .001), whereas presence of superoxide dismutase and inhibition of phosphodiesterase-5 increased cGMP by $46 \% \pm 49 \%$ and $70 \% \pm 77 \%$, respectively $(P<.001)$. In all of the cases EECP increased additional platelet cGMP content, which suggests nitric oxide synthase activation. These acute results showed that very early treatment increases the cGMP production that may participate in the mechanism by which EECP exerts its clinical benefit. Analysis of the modulation of platelet cGMP content suggests that EECP activated the nitric oxide-dependent pathways. (Levenson et al., 2006)

\subsection{Neurohormanal changes}

As with athletic training, the vascular effects of EECP therapy may be mediated through changes in the neurohormonal milieu. Wu et al. showed that EECP therapy has a sustained, dose-related effect in stimulating endothelial cell production of the vasodilator nitric oxide (NO) and in decreasing production of the vasoconstrictor endothelin. (Wu et al. 1999). In another study, Qian et al. showed that the NO level increased linearly in proportion to the hours of EECP treatment.(Qian X et al. 1999). Urano et al. further showed that plasma brain natriuretic peptide levels decreased after EECP therapy and were positively correlated with left ventricular end diastolic pressure and negatively correlated with peak filling rate. They concluded that EECP therapy reduces exercise-induced myocardial ischemia in association with improved left ventricular diastolic filling in patients with CAD. (Urano et al. 2001)

Another possible mechanism explaining EECP's mode of action is that it may affect changes in ventricular function independent of changes in cardiac load. Gorcsan et al. evaluated the effects of EECP therapy on left ventricular function in New York Heart Association class II or III heart failure patients with an EF less than $40 \%$. Their results showed that EECP treatment was associated with improvements in preload adjusted maximal power, a relatively load-independent measure of left ventricular performance and EF, along with a decrease in heart rate in these heart failure patients. (Gorcsan et al. 2000)

\subsection{Anti-inflammatory effect}

A recently published randomized controlled study examining the effect of EECP therapy on inflammatory and adhesion molecules in patients with CAD indicated that EECP therapy has an anti-inflammatory effect in patients with angina pectoris. Patients were randomly assigned to receive active EECP or sham treatment. Plasma tumor necrosis factor- $a$, 
monocyte chemoattractant protein- 1 , and soluble vascular cell adhesion molecule- 1 were measured before and after a full course of 35 1-hour sessions of EECP or sham treatment. Patients in the EECP group demonstrated reductions in tumor necrosis factor-a and monocyte chemoattractant protein-1 after treatment, whereas those in the sham therapy group showed no changes. EECP therapy decreased circulating levels of proinflamatory biomarkers in patients with symptomatic CAD (Casey et al. 2008).

\section{Patient selection}

\subsection{Indications}

FDA Labeled indications for the use of EECP include treatment of patients with:

- Stable or unstable angina pectoris

- Congestive heart failure

- $\quad$ Acute MI

- Cardiogenic shock

\subsection{Which group of patients may benefit from EECP therapy?}

Patients with angina or angina equivalent symptoms such as shortness of breath and/or fatigue who:

- have coronary anatomy unsuitable for surgical or catheter-based revascularization

- $\quad$ inadequately respond to optimum medical therapy

- $\quad$ underwent incomplete invasive revascularization

- $\quad$ are considered inoperable or at high risk of operative/interventional complications

- have comorbid conditions that increase the risk of revascularization procedures, such as diabetes, heart failure, pulmonary disease, and renal dysfunction

- $\quad$ are unwilling to undergo additional invasive revascularization procedures

- have stable (NYHA class II or III) heart failure; patients with any evidence of decompensation should not be treated until they are stable with the use of medical therapy

- have ischemic or idiopathic cardiomyopathy

- have cardiac syndrome X or microvascular ingina

- $\quad$ have LVD $(\mathrm{EF}<35 \%)$

\subsection{Contraindications and side effects}

It is important to point out that EECP therapy is not for everyone. This noninvasive outpatient procedure can be somewhat uncomfortable for patients because of the highpressure sequential compression of the cuffs. It is not recommended for certain types of valvular heart disease (especially aortic insufficiency), or for those with recent cardiac catheterization, an irregular heart rhythm, severe hypertension, significant blockages in the leg arteries, or a history of deep venous thrombosis. For anyone else, however, the procedure seems to be quite safe.

\subsection{Contraindications}

- Moderate to severe aortic insufficiency (regurgitation would prevent diastolic augmentation)

- Arrhythmias that may interfere with EECP system triggering (uncontrolled atrial fibrillation or fl utter or very frequent premature ventricular contractions) 
- $\quad$ Coagulopathy with an INR of prothrombin time greater than 2.5

- Severe hypertension: greater than $180 / 110 \mathrm{~mm} \mathrm{Hg}$ (the augmented diastolic pressure may exceed safe limits)

- Cardiac catheterization or arterial puncture (risk of bleeding at femoral puncture site) within the past 2 weeks

- Decompensated heart failure

- Severe peripheral arterial disease (reduced vascular volume and muscle mass may prevent active counterpulsation)

- Aortic aneurysm $(\geq 5 \mathrm{~mm}$ ) or dissection (diastolic pressure augmentation may be deleterious)

- Pregnancy or being of childbearing age (effects of EECP therapy on the fetus have not been studied)

- Venous disease (phlebitis, varicose veins, stasis ulcers, prior or current deep vein thrombosis or pulmonary embolism)

- Severe chronic obstructive pulmonary disease (no safety data in pulmonary hypertension)

\subsection{Side effects}

- $\quad$ Skin abrasion or ecchymoses

- Bruises (especially in patients using warfarin in whom the international normalized ratio [INR] is not adjusted)

- Paresthesias

- Leg or waist pain

- Worsening of heart failure in patients with severe arrhythmias

\section{Future research}

Throughout the world, EECP therapy has been studied for various potential uses other than heart disease ((Offergeld et al 2000; Rajaram et al 2006; Hilz et al 2004; Myhre et al 2004).

${ }^{*}$ Cardiac Syndrome $X$ : its role in improving endothelial function might be beneficial in the treatment of patients with Cardiac Syndrome X, which is marked by severe chest pain caused by myocardial dysfunction, often without detectable atherosclerosis. Investigators have reported successful treatment of Cardiac Syndrome $X$ with severely symptomatic coronary endothelial dysfunction in the absence of obstructive CAD with standard 35-h course of EECP therapy (Bonetti et al 2004).

${ }^{*}$ Acute M/Cardiogenic Shock: Based on its acute haemodynamic effects, comparable to those of IABP, EECP can be proposed as a potential treatment for coronary syndromes in acute setting, as an inpatient therapy for patients with IABP contraindications. Recently developed mobile EECP Therapy system, enables its use in the catheter laboratory and operation room settings.

*Erectile dysfunction: Studies looking at EECP therapy and erectile dysfunction have shown a $200 \%$ increase in penile artery flow and reported improvement in erectile function [Froschermaier et al 1998; Lawson et al 2007; El-Sakka AI et al 2007

*Hepatorenal syndrome: Werner et al. assessed the potential role of EECP therapy in diuresis and increased urinary $\mathrm{fl}$ ow in patients with endstage liver disease awaiting a transplant. They found that EECP therapyincreased mean arterial pressure as well as 
urinary production (urinary flow rate) in patients with end-stage cirrhosis and hepatorenal syndrome. (Werner et al 2005. )Studies involving larger sample sizes are necessary to confirm the effectiveness of EECP therapy in erectile dysfunction and hepatorenal syndrome.

* Ischemic stroke: Investigators sought to determine effect of EECP on middle cerebral artery blood flow augmentation in normal controls as a first step to support future clinical trials in acute stroke. (Alexandrow et al.,2008)

* Primary /Secondary Prevention: New studies are under way to explore the role of EECP therapy in primary and secondary prevention of coronary artery disease and its potential use in failing fontan operation.

\section{Conclusion}

Scientific data indicates that EECP provides a safe and effective treatment option for patients with CAD. Its different mode of action complements the invasive revascularization treatment. Currently, The American College of Cardiology/ The American Heart Association recommends EECP Therapy as a Class IIb (Level of Evidence: B) intervention for treatment of CAD. Due to the volume and quality of scientific data now available and per the descriptions of the rating and evidence levels as defined by American College of Cardiology and American Heart Association guidelines it is believed that EECP therapy has earned a Class IIa treatment recommendation with level of evidence A.

\section{References}

American Heart Association. Heart Disease and Stroke Statistics 2010- Update. Amsterdam EA, Banas J, Criley JM, Loeb HS, Mueller H, Willerson JT, Mason DT. 1980. Clinical assessment of external presuure circulatory assistance in acute myocardial infarction. Report of a cooperative clinical trial. Am J Cardiol, 45:349-356.

Alexandrov AW, Ribo M,Wong KS, Sugg RM, Garami Z, Jesurum JT,Montgomery B,Alexandrov A.2008. Perfusion Augmentation in Acute Stroke Using Mechanical Counter-Pulsation-Phase IIa Effect of External Counterpulsation on Middle Cerebral Artery Mean Flow Velocity in Five Healthy Subjects. Stroke,39:2760.) Arora RR, Chou TM, Jain D Fleishman B, Crawford L, McKiernan T, Nesto RW. 1999. The Multicenter Study of Enhanced External Counterpulsation (MUSTEECP):effect of EECP on exercise-induced myocardial ischemia and anginal episodes. J Am Coll Cardiol, 33:1833-1840.

Arora RR, Chou TM, Jain D, Fleishman B, Crawford L, McKiernan T, Nesto R, Ferrans CE, Keller S. 2002. Effects of enhanced external counterpulsation on health-related quality of life continue 12 months after treatment: a substudy of the multicenter study of enhanced external counterpulsation.J Investig Med, 50:25-32.

Bagger JP, Hall RJ, Koutroulis G, Nihoyannopoulos P. 2004. Effect of enhanced external counterpulsation on dobutamine-induced left ventricular wall motion abnormalities in severe chronic angina pectoris. Am J Cardiol, 15;93(4):465-7. 
Birtwell WC, Ruiz U, Soroff HS, DesMarais D, Deterling RA Jr .1986. Technical consideration in the design of a clinical system for external left ventricular assist. Trans Am Soc Artif Intern Organs,14:304-310.

Bonetti PO, Gadasalli SN, Lerman A, Barsness GW. 2004. Successful treatment of symptomatic coronary endothelial dysfunction with enhanced external counterpulsation. Mayo Clin Proc, 79:690 -2

Braverman, DL. 2009. Enhanced external counterpulsation: An innovative physical therapy for refractory angina. $P M E R, 1: 268-76$.

Braith RW, Conti CR, Nichols WW, Choi CY, Khuddus MA, Beck DT, Casey DP. 2010.Enhanced External Counterpulsation Improves Peripheral Artery FlowMediated Dilation in Patients with Chronic Angina. A Randomized ShamControlled Study. Circulation,122:1612-1620.

Cohen LS, Mullins CB, Mithell JH. 1973. Sequenced external counterpulsation and intra aortic balloon pumping in cardiogenic schock. Am J Cardiol,32: 656-661.

Casey DP, Conti CR, Nichols WW, Choi CY, Khuddus MA, Braith RW. 2008. Effect of enhanced external counterpulsation on infl amatory cytokines and adhesion molecules in patients with angina pectoris and angiographic coronary artery disease. Am J Cardiol, 101:300-302.

El-Sakka AI, Morsy AM, Fagih BI, et al.: Enhanced external counterpulsation in patients with coronary artery disease-associated erectile dysfunction. Part II: impact of disease duration and treatment courses. J Sex Med 2007, 4:1448-1453.

Froschermaier SE, Werner D, Leike S, et al.: Enhanced external counterpulsation as a new treatment modality for patients with erectile dysfunction. Urol Int 1998, 61:168-171.

Gorcsan J, Crawford L, Soran OZ. 2000. Improvement in left ventricular performance by enhanced external counterpulsation in patients with heart failure. J Am Coll Cardiol, 35:230A.

Gloekler S, Meier P, Marchi SF, Rutz T, Traupe T, Rimoldi SF, Wustmann K, Steck H, Cook S, Vogel R, Togni M \& Seiler C.2010. Coronary Collateral Growth by External Counterpulsation: A Randomized Controlled Trial. Heart, 96: 202-207

Hilz MJ, Werner D, Marthol H, Flachskampf FA, Daniel WG.Enhanced external counterpulsation improves skin oxygenation and perfusion. Eur J Clin Invest 2004,34:385-91.

Holubkov R, Kennard ED, Foris JM, Kelsey SF, Soran O, Williams DO, Holmes DR.2002. Comparison of Patients Undergoing Enhanced External Counterpulsation and Percutaneous Coronary Intervention for Stable Angina Pectoris. Am J Cardiol,89: 1182-1186.

Katz WE, Gulati V, Feldman AM, Crawford L, Peron M, Soran O, Gorcsan III J. 1998. Effects of enhanced external counter pulsation on internal mammary artery flow; Comparison with intraaortic balloon counterpulsation. J Am Coll Cardiol (Supp A); 31: $85 \mathrm{~A}$.

Lakshmi MV, Kennard ED, Kelsey SF, Holubkov R, Michaels AD.2002. Relation of the pattern of diastolic augmentation during a course of enhanced external counterpulsation (EECP) to clinical benefit (from the International EECP Patient Registry [IEPR]). Am J Cardiol. 89(11):1303-5. 
Lawson WE, Hui JCK, Soroff HS, Zeng SZ, Kayden DS, Sasvary D, Atkins H, Cohn PF.1992. Efficacy of enhanced external counterpulsation in the treatment of angina pectoris. Am J Cardiol, 70:859-862.

Lawson WE, Hui JCK, Cohn PF.2000. Long-term prognosis of patients with angina treated with enhanced external counterpulsation: five-year follow-up study. Clin Cardiol, 23:254-258.

Lawson WE, Pandey K, Hui JCK. 2002. Benefit of enhanced external counterpulsation in coronary patients with left ventricular dysfunction: cardiac or peripheral effect? J CardFail, 8(Suppl 41):146

Lawson WE, Hui JC, Kennard ED.2007. Effect of enhanced external counterpulsation on medically refractory angina patients with erectile dysfunction. Int J Clin Pract, 61:757-762.

Loh PH, Cleland JG, Louis AA, Kennard ED, Cook JF, Caplin JL, Barsness GW, Lawson WE, Soran OZ, Michaels AD.2008. Enhanced external counterpulsation in the treatment of chronic refractory angina: a long-term follow-up outcome from the International Enhanced External Counterpulsation Patient Registry. Clin Cardiol, 31:159-164.

Masuda D, Nohara R, Hirai T, Kataoka K, Chen LG, Hosokawa R, Inubushi M, Tadamura E, Fujita M, Sasayama S. 2001. Enhanced external counterpulsation improved myocardial perfusion and coronary flow reserve in patients with chronic stable angina. Eur Heart J, 22:1451-1458.

Manchanda A, Soran O. 2007.Enhanced external counterpulsation and future directions: step beyond medical management for patients with angina and heart failure. J Am Coll Cardiol. 50:1523-1531.

Masuda D, Nohara R, Kataoka K. 2001. Enhanced external counterpulsation promotes angiogenesis factors in patients with chronic stable angina. Circulation, 104:II445.

Michaels AD, Kennard ED, Kelsey SE, Holubkov R, Soran O, Spence S, Chou TM.2001. Clin Cardiol, Does higher diastolic augmentation predict clinical benefit from enhanced external counterpulsation?: Data from the International EECP Patient Registry (IEPR). 24(6):453-8.

Michaels AD, Accad M, Ports TA, Grossman W. 2002. Left ventricular systolic unloading and augmentation of intracoronary pressure and Doppler fl ow during enhanced external counterpulsation. Circulation,106:1237-1242.

Michaels AD, Tacy T, Teitel D, Shapiro M, Grossman W.2009. Invasive left ventricular energetics during enhanced external counterpulsation. Am J Ther,16(3):239-46.

Myhre LG, Muir I, Schutz RW, Rantala B, Thigpen T. 2004. Enhanced external counterpulsation for improving athletic performance. Paper presented at: Experimental Biology April 17-21, 2004; Washington,DC

Nichols WW, Estrada JC, Braith RW, Owens K, Conti CR.2006. Enhanced external counterpulsation treatment improves arterial wall properties and wave reflection characteristics in patients with refractory angina. J Am Coll Cardiol,19;48(6):1208-14.

Offergeld C, Werner D, Schneider M, Daniel WG, Hüttenbrink KB.Pneumatic external counterpulsation (PECP): a new treatment option in therapy refractory inner ear disorders?2000. Laryngorhinootologie; 79:503-9. 
Pettersson T, Bondesson S, Cojocaru D, Ohlsson O, Wackenfors A, Edvinsson L. 2006. One year follow-up of patients with refractory angina pectoris treated with enhanced external counterpulsation. BMC Cardiovasc Disord , 6:28.

Rajaram SS, Shanahan J, Ash C, Walters AS, Weisfogel G. 2006. Enhanced external counter pulsation (EECP) for restless legs syndrome (RLS): preliminary negative results in a parallel double-blind study. Sleep Med, 7:390 -1.

Soran O, Crawford LE, Schneider VM, Feldman AM. 1999.Enhanced external counterpulsation in the management of patients with cardiovascular disease.Clinical Cardiol,22: 173-178.

Soran O, Michaels AD, Kennard ED, Kelsey SF, Holubkov R, Feldman AM.2001. Is Diastolic Augmentation an Important Predictor of treatment Completion for Patients with Left Ventricular Dysfunction Undergoing enhanced External Counterpulsation for Angina. J. Cardiac Failure,(Suppl 2);7: 99.

Soran O, Kennard ED, Kfoury AG, Kelsey SF; IEPR Investigators. 2006. Two-year clinical outcomes after enhanced external counterpulsation(EECP) therapy in patients with refractory angina pectoris and left ventricular dysfunction (report from The International EECP Patient Registry). Am J Cardiol, 97:17-20.

Soran O, Kennard ED, Kelsey SF, Holubkov R, Strobeck J, Feldman AM. 2002. Enhanced external counterpulsation as treatment for chronic angina in patients with left ventricular dysfunction: a report from the International EECP Patient Registry (IEPR). Congest Heart Fail, 8:297-302.

Soran O, Kennard ED, Bart BA, Kelsey SF; IEPR Investigators.2007. Impact of external counterpulsation treatment on emergency department visits and hospitalizations in refractory angina patients with left ventricular dysfunction. Congest Heart Fail,13:36-40

Soran O, Kennard L, Kelsey SF, On behalf of IEPR Investigators.2010. Comparison of sixmonth clinical outcomes, event free survival rates of patients undergoing enhanced external counterpulsation (EECP) for coronary artery disease in the United States and Europe. European Heart J; (Suppl 31);24:353.

Stys TP, Lawson WE, Hui JCK, Fleishman B, Manzo K, Strobeck JE, Tartaglia J, Ramasamy S, Suwita R, Zeng ZS, Liang H, Wener D. 2002. Effects of enhanced external counterpulsation on stress radionuclide coronary perfusion and exercise capacity in chronic stable angina pectoris. Am J Cardiol. 89:822-882.

Taguchi I, Ogawa K, Kanaya T, Matsuda R, Kuga H, Nakatsugawa M. 2004. Effects of enhanced external counterpulsation on hemodynamics and its mechanism. Circ J,68(11):1030-4.

Urano H, Ikedah H, Ueno T, Matsumoto T, Murohara T, Imaizumi T. 2001. Enhanced external counter pulsation improves exercise tolerance, reduces exercise-induced myocardial ischemia and improves left ventricular diastolic filling in patients with coronary artery disease. J Am Coll Cardiol, 37:93-99.

Qian X, Wu W, Zheng ZS. 1999. Effect of enhanced external counterpulsation on nitric oxide production in coronary disease. J Heart Disease,1:193 
Werner D, Tragner P, Wawer A, et al.: Enhanced external counterpulsation: a new technique to augment renal function in liver cirrhosis. Nephrol Dial Transplant 2005, 20:920926.

Wu GF, Qiang SZ, Zheng ZS. 1999. A neurohormonal mechanism for the effectiveness of the enhanced external counterpulsation. Circulation,100:I832.

Zeng ZS, Li TM, Kambic H, Chen GH, Yu LQ, cai Sr, Zhan CY, Chen YC, Wo SX, Chen GW, Ma H, Chen PJ, Huang BJ, Nose Y. 1983. Sequential external counterpulsation in China. Trans Am Soc Artif Intern Organs,29:593-603. 


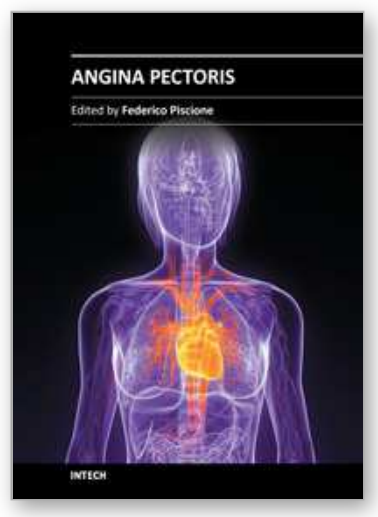

\author{
Angina Pectoris \\ Edited by Prof. Federico Piscione
}

ISBN 978-953-307-359-0

Hard cover, 184 pages

Publisher InTech

Published online 10, October, 2011

Published in print edition October, 2011

Angina is the most common disorder affecting patients with ischemic heart disease. This book provides a thorough review of fundamental principles of diagnosis, pathophysiology and treatment of angina pectoris, representing an invaluable resource not only for cardiologists, but also for general practitioners and medical students.

\title{
How to reference
}

In order to correctly reference this scholarly work, feel free to copy and paste the following:

Ozlem Soran (2011). The Role of Enhanced External Counterpulsation Therapy in the Management of Coronary Artery Disease, Angina Pectoris, Prof. Federico Piscione (Ed.), ISBN: 978-953-307-359-0, InTech, Available from: http://www.intechopen.com/books/angina-pectoris/the-role-of-enhanced-externalcounterpulsation-therapy-in-the-management-of-coronary-artery-disease

\section{INTECH}

open science | open minds

\author{
InTech Europe \\ University Campus STeP Ri \\ Slavka Krautzeka 83/A \\ 51000 Rijeka, Croatia \\ Phone: +385 (51) 770447 \\ Fax: +385 (51) 686166 \\ www.intechopen.com
}

\author{
InTech China \\ Unit 405, Office Block, Hotel Equatorial Shanghai \\ No.65, Yan An Road (West), Shanghai, 200040, China \\ 中国上海市延安西路65号上海国际贵都大饭店办公楼405单元 \\ Phone: +86-21-62489820 \\ Fax: +86-21-62489821
}


(C) 2011 The Author(s). Licensee IntechOpen. This is an open access article distributed under the terms of the Creative Commons Attribution 3.0 License, which permits unrestricted use, distribution, and reproduction in any medium, provided the original work is properly cited. 